Uberschusses an Kalkwasser keine einheitlichen sicheren Werte orhalten werden, da mit steigendem Zusatz von Kalk steigende Mengen dieses Körpers nit dem ausfallendem Carbonat mit niedergerisen werden, wie dies Tillmans und Heublein (Z. Nahr.- u. GenuBm. 33, 289-304 [1917]; Angew. Chem. 30, II, 409 [1917]) bei Verwendung von Ätzbaryt gemäß dem Petten. k of er-Trillich schen. Verfahren ausführlich nachgewiesen haben. Ganz ähnlich tritt ein solcher Mehrverbrauch an Kalkwasser, wie sich Vf. überzeugt hat, ein, je gröBer der Zusatz an Kalkwasser bei dem Versuche ist. Von dieser Unsicherheit kann man sich aber fernhalten und der Berechnung des erforderlichen Kalkzusatzes eine Bestimmung der freien Kohlensäure nach denı Trilli c h schen Verfahren in seiner Neugestaltung durch Till. m a n s (Z. Nahr.- u. Genußm. 33, 299 [1917]) und der Carbonatkohlensäure nuch dem üblichen allgemein anerkannten Verfahren durch Titration mit $1 / 10^{-n}$. Süure unter Anwendung von Methylorange als Indicator zugrunde legen.

Zum Schlusse möge noch erwähnt werden, daB nach den auf breiter Basis ausgeführten Versuchen von Tillmans und $\mathrm{H}$ e u b le in (Gesundh.-Ing. 35, 669 [1912]) alle Wässer, welche Bicarbonate enthalten, auch eine gewisse über die Bicarbonatkohlensăure hinausgehende Menge an überschüssiger (also theoretisch gesprochen freier) Kohlensäure enthalten mü s s e n. Dieso Menge an überschüssiger freier Kohlensäure steigt mit höherem Gehalt des Wassers an gelöstem Bicarbonat in gesetzmåigem Verhaltnis. Daraus geht wohl ohne weiteres die Notwendigkeit der Berücksichtigung der freien Kohlensäure bei der Wasserreinigung hervor, obwohl zugegeben sein mag, daß dieser Punkt auch in den meisten Lehrbüchern übersehen ist. Gerade deshalb collte bei dieser Gelegenheit einmal nachdrücklich darauf hingewiesen werden.

Zu Vorstehendem bemerkt Herr Prof. Dr. H. Noll folgendes:

Vikt o r R od $t$ weist darauf hin, daß ich bei der Berechnung der erforderlichen Zusătze die freie Kohlensäure unberücksichtigt gelassen hätte, und dadurch eine richtige Enthärtung des Wassers unmöglich wäre. Die Antwort darauf ist eigentlich schon in meiner Veröffentlichung gegeben, in welcher ich unter Bezugnahme auf das D rawe sohe Verfahren (Angew. Chem. 23, 52 [1910]), bei welchem die zur Füllung von Eisen, Aluminium, Mangan, organischen Stoffen oder Kieselsäure, sowie die zur Neutralisation freier organisoher und anorganischer $\mathrm{S}$ a u r e n erforderlichen Mengen an Zusatzatoffen berücksichtigt werden, ausdrücklich darauf hingewiesen habe, $\mathrm{daB}$, wenn sich diese Stoffe einmal in größerer Menge im Wasser vorfinden sollten, die Enthärtung des Wassers dadurch ungünstig beeinflußt werden könnte. Solche Fälle würden sich aber bei der Kontrolle des Wassers bemerkbar machen und würden dadurch eliminiert werden können, daß man beim Kalk-Sodaverfahren den Kalkzusatz und boim KalkNatriumhydroxydverfahren bei den Beispielen I und II den Kalk. zusatz und bei den Beispielen III und IV den Natriumhydroxyd. zusatz versuchsweise um einen Grad erhöhte. Man würde dann bald sehen, ob man zu wenig oder zu viel zugesetzt hätte und danach einen Durchschnittswert festlegen können. Handele es sich um ganz anormale Wässer, so würde sich das Heranziehen eines Chemikers empfehlen. Die Trilli o h sche Methode ist von mir absichtlich nicht mit in Vorschlag gebracht worden, um die chemischen Bestimmungen auf das möglichste einzuschränken. Liegen nicht ganz anormale Verhältnisse vor, so wird sich der Nichtchemiker bei der Enthärtung von Wässern, die reichliche Mengen an Kohlensäure enthalten, in der von mir angegebenen Weise helfen können. Bei Wässern, die sich nur unwesentlich verändern, wird das wenig Mähe machen, da Kontrollen nur selten wiederholt zu werden brauchen, und bei Oberflächenwüssern, die in bezug auf ihre Zusammensetzung größeren Schwankungen unterworfen sind und infolgedessen ständig kontrolliert werden müssen, ist der Kohlensäuregehalt in der Regel so gering, $\operatorname{da} \beta$ er ganz wird vernachlässigt werden können. Dabei würde noch $\mathrm{zu}$ berücksichtigen sein, $\mathrm{daB}$ die von mir empfohlene überschüssig zugesetzte Soda sowieso der Bildung von Bicarbonaten der Härtebildner entgegenwirkt.

$R$ o d t führt in seinem Artikel dann noch aus, daB für die Berechnung der erforderlichen Zusatze an Kalkmilch nicht die Ermittlung der Carbonathärte genügte, sondern die Bestimmung der freien und halbgebundenen Kohlensiiure erforderlich wäre. Ich verstehe diese Auffassung nicht, da aus der Carbonathärte ja dio zur Abbindung der halbgebundenen Kohlensäure erforderlichen Kalk. mengen berechnet werden. Die gebundene Kohlensäure, die sich bei der Bestimmung der Carbonathärte ergibt, ist der halbgebundenen Kohlensalure stets gleich, wenn die Wässer freie Kohlensäure enthalten, was praktisch immer der Fall ist. Außer der Carbo. nathärte muß man aber die Gesamtmagnesiummenge kennen, un die Kalkmengen für Magnesiummonocarbonat und Chlormagnesium berechnen zu können.

Das Niederreißen von Kalk durch die Carbonate, was Rod t noch erwähnt, dürfte für die Wasserreinigungsfrage von nebensäch. licher Bedeutung sein. Beim Hamburger Leitungswasser habe ich vergleichende Untersuchungen nach dem $D$ r a w e schen und nach dem von mir vorgeschlagenen Verfahren angestellt und bin dabei zu sehr gut übereinstimmenden und brauchbaren Resultaten gekommen. Für die Enthärtung des Wassers ist das einfachste und am schnellsten ausführbare Verfahren das beste, zumal für die Nicht. chemiker, da diesen dadurch Gelegenheit gegeben ist, sich von fremder Hilfe unabhängig machen zu können.

[Zu A. 13i].

\section{Eine einfache Vorrichtung zum gleichzeitigen Rühren und Filtrieren.}

Von Ing.-Chem. Fritz Feior, dzt. im Felde.

Aus dem chemischen Laboratorium des Vereins „Volksheim" Wien.

Versucht man, die Löslichkeitsverhältnisse einer Verbindung oder eines Salzgemenges bei höherer 'Temperatur dadurch zu bestimmen, daß man aliquote Teilo der Flüssigkeit außerhalb des Thermostaten durch ein Warmwasserfilter usw. filtriert und im Filtrat die Konzentration des Gelösten ermittelt, so erhält man meist falsche Resultate.

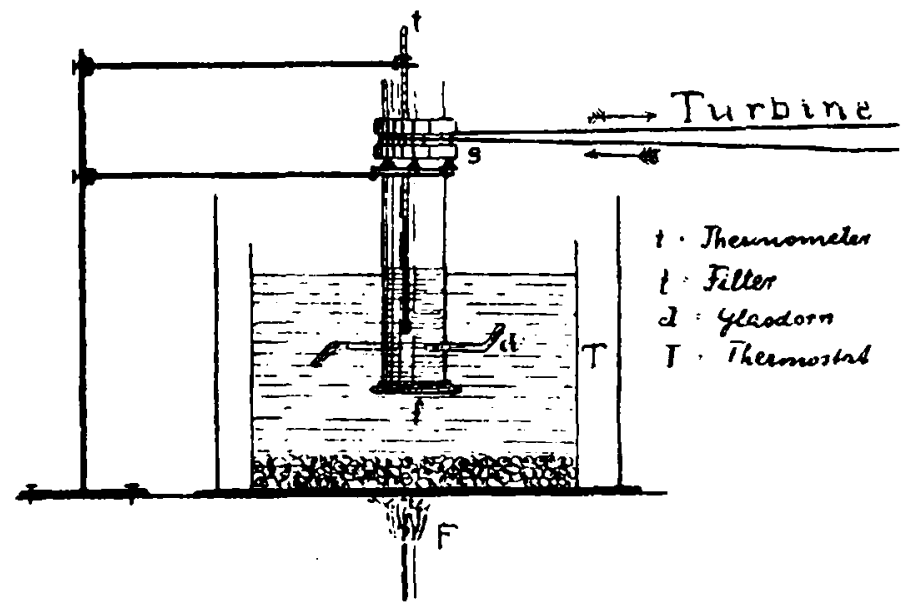

Abgesehen von der Schwierigkeit des Gleichhaltens der Tempcratur, stört stets die Absorptionwirkung des Filters.

Folgende einfache Vorrichtung (vgl. Abbiddung) ermöglicht die Entuahme einer vom Bodenkörper völlig freien Probe direkt aus dem Thermostaten.

Ein unten umgestülptes Glasrobr von geeigneter Weite (etwa Hartglaseprouvette) wird mit einem Mullstück oder gehärtetem Filtrierpapier abgeschlossen und in einem Holzring befestigt, der zum Antrieb durch eine Turbine eine seitliche Rille trägt. -- Durch Anschmelzen mehrerer Glasdorne wirkt das Rohr gleichzeitig als Rübrer.

Bei Anwendung geeigneten Filtermaterials ist die Flüssigkeit im Glasrohr völlig rein, und zar Analyse kann direkt aus demselben pipettiert werden.

Verwendet man statt eines Filters eine halbdurchlässige Membran, so eignet sich dieselbe Vorrichtung für Bestimmungen der Diffusionsgeschwindigkeiten bei Kolloiden unterhalb deren Koagulationstemperatur.
[A. 144.] 